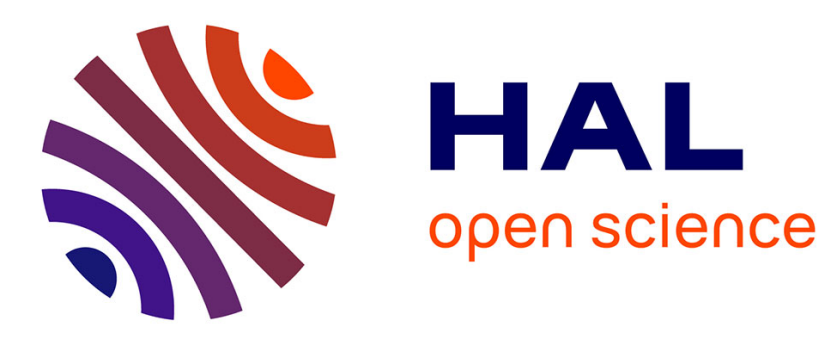

\title{
Inertial Velocity Estimation for Indoor Navigation Through Magnetic Gradient-based EKF and LSTM Learning Model
}

\author{
Makia Zmitri, Hassen Fourati, Christophe Prieur
}

\section{To cite this version:}

Makia Zmitri, Hassen Fourati, Christophe Prieur. Inertial Velocity Estimation for Indoor Navigation Through Magnetic Gradient-based EKF and LSTM Learning Model. IROS 2020 - IEEE/RSJ International Conference on Intelligent Robots and Systems, Oct 2020, Las Vegas, United States. pp.1-6, 10.1109/IROS45743.2020.9340772 . hal-02958106

\author{
HAL Id: hal-02958106 \\ https://hal.science/hal-02958106
}

Submitted on 5 Oct 2020

HAL is a multi-disciplinary open access archive for the deposit and dissemination of scientific research documents, whether they are published or not. The documents may come from teaching and research institutions in France or abroad, or from public or private research centers.
L'archive ouverte pluridisciplinaire HAL, est destinée au dépôt et à la diffusion de documents scientifiques de niveau recherche, publiés ou non, émanant des établissements d'enseignement et de recherche français ou étrangers, des laboratoires publics ou privés. 


\title{
Inertial Velocity Estimation for Indoor Navigation Through Magnetic Gradient-based EKF and LSTM Learning Model
}

\author{
Makia Zmitri, Hassen Fourati and Christophe Prieur
}

\begin{abstract}
This paper presents a novel method to improve the inertial velocity estimation of a mobile body, for indoor navigation, using solely raw data from a triad of inertial sensors (accelerometer and gyroscope), as well as a determined arrangement of magnetometers array. The key idea of the method is the use of deep neural networks to dynamically tune the measurement covariance matrix of an Extended Kalman Filter (EKF). To do so, a Long Short-Term Memory (LSTM) model is derived to determine a pseudo-measurement of inertial velocity of the target under investigation. This measurement is used afterwords to dynamically adapt the measurement noise parameters of a magnetic field gradient-based EKF. As it was shown in the literature, there is a strong relation between inertial velocity and magnetic field gradient, which is highlighted with the proposed approach in this paper. Its performance is tested on the Openshoe dataset, and the obtained results compete with the INS/ZUPT approach, that unlike the proposed solution, can only be applied on foot-mounted applications and is not adequate to all walking paces.
\end{abstract}

\section{INTRODUCTION}

The recent growth of interest in smart systems technology, autonomous robotics and location-aware services generates a strong motivation to develop robust and efficient techniques for tracking the position of a moving body, in both outdoor and indoor environments. Indoor navigation is difficult to deal with because of the limited indoor coverage of Global Positioning System (GPS). Nowadays, different solutions have been proposed to deal with this issue, most of them use a variety of sensors that usually require costly and pre-installed infrastructures, which can be inaccessible in various applications that introduce hostile environments or emergency situations. For this reason, navigation solutions that are independent from pre-equipped buildings are highly preferred. The most used technique in these situations is the one fusing data from low-cost Inertial Measurement Units (IMUs), composed of accelerometers and gyroscopes. Different approaches have been proposed to effectively handle these sensors, as they introduce biases and errors, and a simple integration of their outputs can not provide any accurate position or orientation (attitude) estimations [11]. One possible solution is foot-mounted dead-reckoning using the Zero-Velocity Update Technique (ZUPT) [5]. However, this approach needs a very specific parameters tuning that depends on the nature of the walking activity, which makes the ZUPT unsuitable to general cases. Another way of exploiting IMUs is by combining them with magnetic sensors. In fact,

All authors are with Univ. Grenoble Alpes, CNRS, Grenoble INP, GIPSAlab, F-38000 Grenoble, France.

This work has been partially supported by MIAI@Grenoble Alpes, (ANR-19-P3IA-0003). in indoor environments, important magnetic perturbations are observed [2], that hold valuable information for indoor localization. One of the innovative techniques that takes advantage of these perturbations, has been firstly introduced in [15] and [16]. It states that as long as the magnetic field gradient is non-singular, the velocity can be reconstructed, from only a 3-axis magnetometers array, a 3 -axis accelerometer and a 3-axis gyroscope measurements. This innovative technique can be applied on any moving object in indoor environments, and doesn't require any particular sensors location. In [15], authors considered that the magnetic field gradient is unknown and assumed that it satisfies some first order dynamics driven by white noises. In [16], the same authors extended their approach by measuring the magnetic field gradient (using three 3-axis magnetometers) along side with the previous assumption (white noise-based dynamic equation). To the best of the authors knowledge, this choice is not fit to capture the magnetic field gradient dynamics and can consequently degrade velocity estimation. For this reason, in [18], a magnetic field gradient-based Extended Kalman Filter (EKF) is proposed, that better models the magnetic field gradient dynamics by using a set of spatially distributed magnetometers array, and as a result, velocity estimation is more accurate. One of the well-known difficulties of Kalman filtering is its covariance matrices tuning (both process $Q$ and measurement $N$ covariances). Not providing the adequate values to the different noise parameters in these matrices causes unbounded errors and thus, unexpected estimation results. In fact, there is no general solution to properly tune these covariance matrices and usually, it is based on empirical methods. More particularly, giving fixed noise parameters to the measurement covariance $N$ is unsuitable, as the errors in sensor measurements vary in time, due to different external factors (e.g temperature). In [10], different tuning methodologies were demonstrated, to accurately handle an EKF-based INS/GPS fusion. In [3], authors used a CNN to tune the noise parameters of an Invariant Extended Kalman Filter (IEKF), for the dead-reckoning of wheeled vehicles. This was done by exploiting the assumptions on the velocity that were presented in the form of pseudo-measurements, and controlling their corresponding covariance matrices.

On the basis of this idea, an original architecture is proposed in this paper to improve the performance of the magnetic field gradient-based EKF. The approach dynamically adapts the measurement covariance matrix $N$ of the EKF, using deep learning networks, in the purpose of having a better inertial velocity estimation. To do so, a LSTM model is trained to compute a pseudo-measurement of inertial velocity. The 
latter is then fed to the EKF and used to properly set the degree of confidence given to it by the filter. This is encoded in its corresponding covariance matrix that is set automatically by the proposed adapter. To evaluate the performance of the proposed approach, a comparison is achieved with the traditional INS/ZUPT method on the open source Openshoe dataset [14]. First, a notable improvement on the velocity estimation is displayed, compared to when only the magnetic field gradient-based EKF with fixed covariance matrices is used. Then, the estimation results of the proposed approach are shown to be very close to the INS/ZUPT results. This is considered very promising, having in mind that unlike ZUPT, the proposed method is independent from walking paces (i.e. walking slowly or running) and is applicable on any other kind of dataset, which doesn't have to correspond to a foot-mounted framework, and can be extended to other applications.

\section{PRoblem Statement}

The proposed work in this paper is related to the navigation of a rigid body in indoor environments, by relying only on measurements from Micro Electro Mechanical Systems (MEMS) inertial sensors, as well as a spatially distributed 3-axis MEMS magnetometer's array. The goal is to design an approach that estimates the attitude matrix $R \in S O(3)$ (the group of rotations in $3 D$ Euclidean space), the inertial velocity $v_{n}=\left[\begin{array}{lll}v_{n x} & v_{n y} & v_{n z}\end{array}\right]^{\top} \in \mathbb{R}^{3 \times 1}$ and eventually if possible the position $M_{n}=\left[\begin{array}{lll}M_{n x} & M_{n y} & M_{n z}\end{array}\right]^{\top} \in \mathbb{R}^{3 \times 1}$ from a given starting configuration $\left(R_{0}, v_{0}, M_{0}\right)$.

\section{A. Equations of motion}

Two frames of coordinates are used to deal with the problem cited above:

- a local inertial frame $\Re_{n}$ fixed to the Earth and its associated orthonormal basis $\mathfrak{B}_{\mathfrak{n}}=\left(\vec{i}_{n}, \vec{j}_{n}, \vec{k}_{n}\right)$;

- a body frame $\Re_{b}$ attached to the moving body and its associated orthonormal basis $\mathfrak{B}_{\mathfrak{b}}=\left(\vec{i}_{b}, \vec{j}_{b}, \vec{k}_{b}\right)$.

Variables expressed in $\Re_{n}$ (resp. $\Re_{b}$ ) are marked by the subscript $n$ (resp. b).

The attitude matrix $R$ represents the rotation between these two frames, from $\Re_{n}$ to $\Re_{b}$. This matrix can be expressed in terms of quaternion, denoted by $q$, such as $R=R(q)$ [18]. The quaternion is a hypercomplex number of rank 4 such that $q=\left[\begin{array}{ll}q_{0} & q_{v e c t}^{\top}\end{array}\right]^{\top}$, where $q_{0}$ is the scalar part and $q_{\text {vect }}=\left[\begin{array}{lll}q_{1} & q_{2} & q_{3}\end{array}\right]^{\top}$ is the vector part. The reader is invited to refer to [9] for more details about quaternion algebra.

The main equation that describes the variation of quaternion, can be defined from angular velocity measurements $\omega=$ $\left[\begin{array}{lll}\omega_{x} & \omega_{y} & \omega_{z}\end{array}\right]^{\top} \in \mathbb{R}^{3 \times 1}$ of $\Re_{b}$ with respect to $\Re_{n}$, given by a 3-axis gyroscope such as,

$$
\frac{d q}{d t}=\frac{1}{2}\left[\omega_{q} \times\right] q
$$

where $\omega_{q}=\left[\begin{array}{ll}0 & \omega^{\top}\end{array}\right]^{\top}$ and $\left[\omega_{q} \times\right]$ is its skew-symmetric matrix.
To determine the inertial velocity $v_{n}$ and position $M_{n}$, the following kinematic model is introduced,

$$
\frac{d v_{n}}{d t}=R^{\top} a_{b}-g \quad ; \quad \frac{d M_{n}}{d t}=v_{n}
$$

where $a_{b}=\left[\begin{array}{lll}a_{b x} & a_{b y} & a_{b z}\end{array}\right]^{\top} \in \mathbb{R}^{3 \times 1}$ is the acceleration in $\Re_{b}$, measured by a 3-axis accelerometer. In this work the effects of Earth rotation and Coriolis acceleration are ignored, and the gravity vector $g=\left[\begin{array}{lll}0 & 0 & g_{z}\end{array}\right]^{\top} \in \mathbb{R}^{3 \times 1}$ is considered known.

\section{B. The magnetic field gradient to observe velocity}

The following variables can be introduced at first:

- The magnetic field measured in $\Re_{b}$ by a 3-axis magnetometer, which depends on time and space, $B_{b}=$ $\left[\begin{array}{lll}B_{b x} & B_{b y} & B_{b z}\end{array}\right]^{\top} \in \mathbb{R}^{3 \times 1}$;

- The Jacobian matrix $\nabla B_{b} \in \mathbb{R}^{3 \times 3}$, which represents the gradient of the magnetic field, measured on a fixed point $P_{b}=\left[\begin{array}{lll}x_{b p} & y_{b p} & z_{b p}\end{array}\right]^{\top} \in \mathbb{R}^{3 \times 1}$ and defined by

$$
\nabla B_{b}\left(P_{b}(t)\right)=\frac{\partial B_{b}\left(P_{b}(t)\right)}{\partial P_{b}(t)}
$$

In this work, the magnetic field disturbances, that are often observed indoors [2], are used to generate accurate estimates of velocity. This technique was firstly introduced in [15], and it resides on deriving Maxwell's equations [7] to obtain the following dynamic equation ${ }^{1}$ of the sensed magnetic field in $\Re_{b}$,

$$
\frac{d B_{b}}{d t}=-\omega \times B_{b}+\nabla B_{b} v_{b}
$$

where $v_{b}=R v_{n} \in \mathbb{R}^{3 \times 1}$ is the velocity in $\Re_{b}$.

As long as $\nabla B_{b}$ is non-singular, $v_{b}$ is observable and can be estimated (see [16] for the observability proof). However, a remaining issue in this case is to reliably measure $\nabla B_{b}$. In [15] and [16], model (4) is considered and $\nabla B_{b}$ measurements are computed from a magnetometers array and assumed to satisfy a white noise-based model. In this case, the following points can be mentioned:

- the obtained $\nabla B_{b}$ from the magnetometers array may contain a large noise coming first, from each noisy magnetometer measurement, and second, from the approximations taken into account to compute the different Jacobians representing the elements of $\nabla B_{b}$;

- modeling $\nabla B_{b}$ components with a white noise is not sufficient to capture full information on their dynamics.

These two arguments suggest that an accurate representation of $\nabla B_{b}$ needs to be derived, or else, velocity estimation will suffer from unbounded errors especially when $\nabla B_{b}$ has low values, as shown in [4].

In [18], a novel method that deals with this issue is proposed, through introducing a new equation that models efficiently the dynamics of $\nabla B_{b}$, using higher order spatial derivatives of the magnetic field,

$$
\frac{d \nabla B_{b}}{d t}=T_{b} v_{b}+\nabla B_{b}[\omega \times]-[\omega \times] \nabla B_{b}
$$

${ }^{1} \times$ is the cross product of two vectors in $\mathbb{R}^{3}$. 
where $T_{b} \in \mathbb{R}^{3 \times 3 \times 3}$ is a tensor representing the first spatial derivative of $\nabla B_{b}$ in $\Re_{b}$. The reader can refer to [18] for more information on how $T_{b}$ is measured.

\section{Continuous-time dynamic model}

To estimate attitude, inertial velocity and eventually position, a magnetic field gradient-based EKF alongside with a LSTM model are proposed. The EKF is established using the continuous-time dynamic model represented as follows:

$$
\left\{\begin{array}{l}
\frac{d q}{d t}=\frac{1}{2}\left[\omega_{q} \times\right] q \\
\frac{d v_{n}}{d t}=R^{\top} a_{b}-g \\
\frac{d B_{b}}{d t}=-\omega \times B_{b}+\nabla B_{b} R v_{n} \\
\frac{d \nabla B_{b}}{d t}=T_{b} R v_{n}+\nabla B_{b}[\omega \times]-[\omega \times] \nabla B_{b}
\end{array}\right.
$$

The state vector for this dynamic model is $x=$ $\left[\begin{array}{llll}q & v_{n} & B_{b} & \nabla B_{b}\end{array}\right]^{\top} \in \mathbb{R}^{15 \times 1}$, the input vector is $u=$ $\left[\begin{array}{lll}\omega & a_{b} & T_{b}\end{array}\right]^{\top} \in \mathbb{R}^{13 \times 1}$, and the output (measurement) vector is $y=\left[\begin{array}{ll}B_{b} & \nabla B_{b}\end{array}\right]^{\top} \in \mathbb{R}^{8 \times 1}$. Recall that only 7 elements of $T_{b}$ are sufficient to calculate all the tensor's components, see [18]. This model is used to design the magnetic field gradient-based EKF, however, minor changes are applied when combining the latter with a LSTM model, in particular, a new element is added to the measurement vector. This proposed EKF-LSTM estimation approach is explained further in the next section.

\section{EKF-LSTM ESTIMATION APPROACH}

In this section, the proposed estimation schema is detailed. A LSTM-based learning model is used to dynamically tune the measurement covariance matrix $N \in \mathbb{R}^{11 \times 11}$ of the magnetic field gradient-based EKF. The approach which consists of three main blocks, is represented in Fig. 1. and is summarized as follows:

- a LSTM-model is trained to give a pseudomeasurement of inertial velocity, denoted $v_{l s t m}=\left[\begin{array}{lll}v_{n x, l s t m} & v_{n y, l s t m} & v_{n z, l s t m}\end{array}\right]^{\top} \in \mathbb{R}^{3 \times 1} ;$

- $v_{l s t m}$ is added to the output vector of the magnetic field gradient-based EKF, represented by the continuous-time dynamic model (6), such as $y=\left[\begin{array}{lll}B_{b} & \nabla B_{b} & v_{l s t m}\end{array}\right]^{\top} \in$ $\mathbb{R}^{11 \times 1}$;

- the measurement covariance matrix adapter determines the most suitable noise parameters of the measurement covariance matrix $N$ corresponding to $v_{l s t m}$;

- EKF uses the continuous-time dynamic model (6), inertial and magnetic measurements, $v_{l s t m}$ and $N$ to better estimate the states, especially the magnetic field gradient and the velocity.

In the following subsections a detailed explanation of the two blocks: the LSTM model and the measurement covariance matrix adapter, used in the magnetic field gradient basedEKF, to estimate $\widehat{x}=\left[\begin{array}{llll}\widehat{q} & \widehat{v_{n}} & \widehat{B_{b}} & \widehat{\nabla B_{b}}\end{array}\right]^{\top}$.

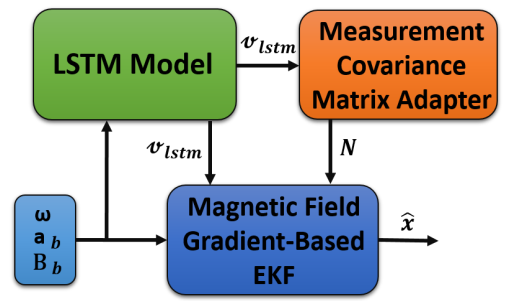

Fig. 1: The proposed EKF-LSTM estimation system

\section{A. LSTM model}

The use of a deep learning algorithm inside the proposed approach is done to obtain a pseudo-measurement of inertial velocity. This pseudo-measurement is then used in the EKF, and the level of confidence given to this measurement is controlled by $N$, as it will be explained further in the next subsection.

After reviewing the literature, it appears that the LSTM is one of the best learning solutions. This choice can actually be straightforward as LSTM-based models are considered very suitable for time series data, and they do not require any complex implementation. LSTM is an extension of artificial Recurrent Neural Network (RNN) architecture [6]. Its particularity is that it has feedback connections, which distinguish it from standard feedforward neural networks [12]. These feedback connections qualify the network to not only process single data points, but also an entire sequence of data, by retaining data information over long time periods.

\section{B. Measurement covariance matrix adapter}

The pseudo-measurement of inertial velocity $v_{l s t m}$ is fed from the trained LSTM-based model to the magnetic field gradient-based EKF. At this point, the role of the measurement covariance matrix adapter, represented with the orange block in Fig. 1. is to dynamically assess the noise parameters in $N$, that reflect confidence in the new measurement $v_{l s t m}$. The measurement covariance matrix can be represented with $\mathrm{N}=\operatorname{diag}\left(\mathrm{N}_{1}, \mathrm{~N}_{2}, \mathrm{~N}_{3}\right)$, where $N_{1<i \leq 3}$ are the measurement covariance matrices corresponding to $B_{b}, \nabla B_{b}$ and $v_{l s t m}$ respectively. In this work, the main interest is in $N_{3} \in \mathbb{R}^{3 \times 3}$, the covariance matrix of the measurement $v_{\text {lstm }}$. Regarding the other noise parameters (i.e. $N_{1<i \leq 2}$ ), their values are fixed taking into account the noise standard deviation, indicated in the magnetometer datasheet, and their dynamic adaptation is left for future work. The same goes for the process covariance matrix $Q \in \mathbb{R}^{15 \times 15}$.

Algorithm 1 represents the measurement covariance matrix adapter, with $\alpha \in \mathbb{R}_{>0}$ a multiplication factor, and $\epsilon \in \mathbb{R}_{>0}$ a chosen threshold that indicates whether the amplitude of the LSTM predicted velocity is sufficiently low.

Then, the EKF estimates $v_{n}$ using the magnetic field gradient-based technique, aided by the LSTM estimate $v_{l s t m}$. This is clearly understood when large values are assigned (equal to $\alpha \mathbb{I}_{3}$ ) to $N_{3, i}$. On the other hand, when $v_{l s t m}$ has an amplitude that is close to zero, the EKF gives it more 


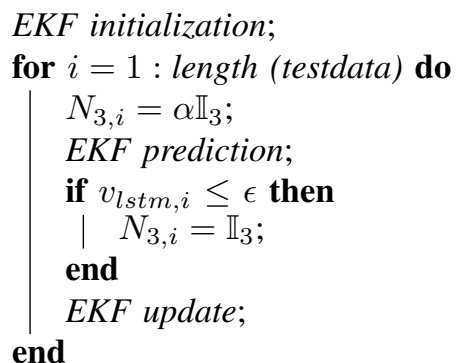

Algorithm 1: Measurement covariance matrix adapter

confidence by attributing lower values (equal to $\mathbb{I}_{3}$ ) to $N_{3, i}$. The reason for this strategy is the fact that $v_{l s t m}$ prediction is more accurate when its 3-axis values are close to zero, than when they are not. This behavior can be explained with the insufficiency of data that corresponds to high amplitudes of inertial velocity in the used training dataset. To back up this assumption, the impact of the high amplitudes training data percentage on the predicted velocity $v_{l s t m}$ accuracy is analyzed using the boxplot [17] in Fig. 2.

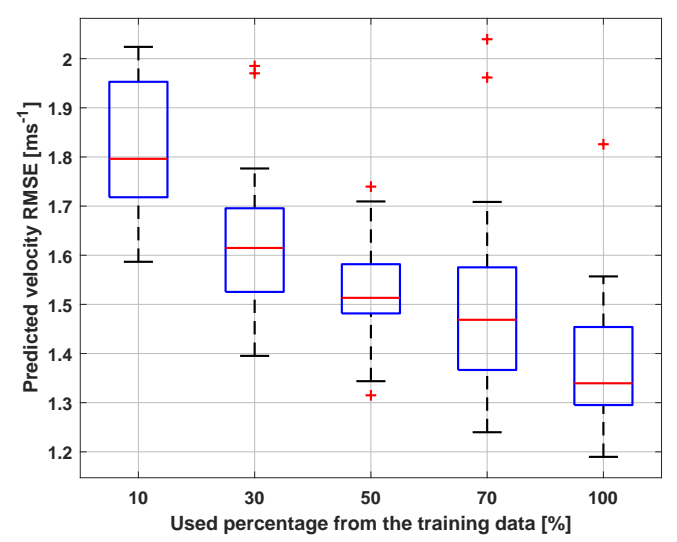

Fig. 2: Effect of high amplitudes training data percentage on RMSE of predicted velocity. Larger the percentage of training data, the smaller velocity RMSE of the testing data becomes.

For this experiment, a dataset with 5913 samples is used. It is created from the Openshoe dataset and contains sensor measurements corresponding to dynamic phases with high velocity amplitudes. This dataset is divided into $70 \%$ (equivalent to 4139 samples) for the training, and $30 \%$ (equivalent to 1774 samples) for the testing. Then the Root Mean Square Error (RMSE) between the INS/ZUPT-based velocity and the LSTM predicted one (of the testing set) is measured for different used percentages of the training dataset. The process is repeated 20 times for each training data percentage. Note that the $30 \%$ corresponding to the testing set are always fixed. As the training data percentage increases, the RMSE of the predicted velocity of the testing set gradually decreases (for instance by looking at the median values represented by the red horizontal lines). This result indicates that as more inputs are provided to the LSTM during training for the dynamic phases, the prediction of $v_{l s t m}$ is more accurate.

Nevertheless, high accuracy of the deep learning network is not mandatory in this work. A small training database is sufficient to detect when $v_{l s t m}$ is close to zero. The low accuracy during the dynamic phases is handled through the tuning of the magnetic field gradient-based EKF with the measurement covariance matrix adapter.

\section{EXPERIMENTAL SCENARIO AND RESULTS}

The performance of the magnetic field gradient-based EKF is presented in this section, and how the inertial velocity estimation results should be improved. Then, the proposed EKF-LSTM approach is applied and it exhibits better performance on the velocity estimation and it impacts the position determination.

\section{A. Magnetic field gradient-based EKF}

In order to assess the performance of the magnetic field gradient-based EKF, $50 \%$ of the data provided by Openshoe is used ${ }^{2}$ Under Matlab, a comparison between the proposed algorithm, and the INS/ZUPT-based velocity estimation [13] is achieved. In Fig. 3, the estimation results for $v_{n}$ are plotted. The proposed EKF succeeds to estimate accurate inertial velocity at some phases of the walking scenario, without any additional velocity correction as the case for the INS/ZUPTbased method.
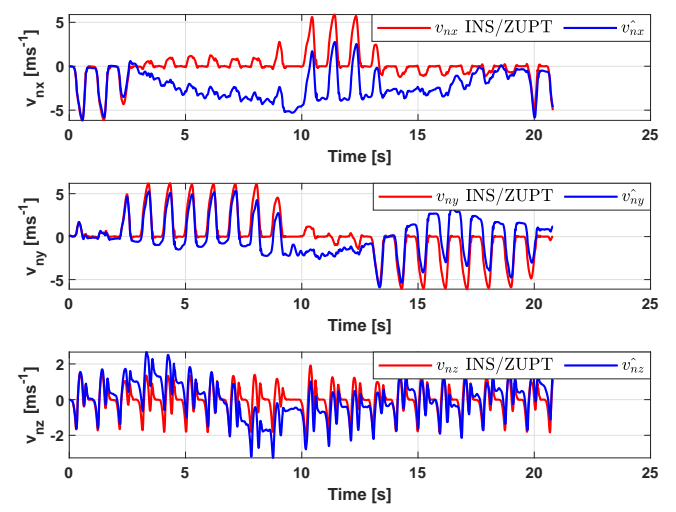

Fig. 3: Estimation of $v_{n}$ with the magnetic field gradientbased EKF and without the LSTM algorithm

Despite the intermittent errors that are seen in the comparison above, this first result is very promising, as it doesn't rely on any foot-mounted related technique, and is independent from the walking pace, as the case for ZUPT. To the best of authors knowledge, the proposed method can be extended to any other type of applications (drones, wheeled-robots, etc.), that are in search for reliable methods for inertial velocity estimation.

There are multiple sources of errors that are seen in Fig. 3 . For instance, the different approximations taken into account to extract the spatial derivatives of $\nabla B_{b}\left(T_{b}\right.$ for example) can

\footnotetext{
${ }^{2}$ The dataset provides measurements for only one 3 -axis magnetometer. The rest of the magnetic array data is simulated.
} 
lead to some errors, others can be the result of linearization process of the EKF, etc.

One of the additional encountered difficulties when dealing with the Kalman filter, is the tuning of its covariance matrices (i.e. process and measurement covariances). Then, using a fixed $N$, is not suitable for situations where the error in sensor readings varies in time due to external factors (e.g. temperature). Based on this idea, an EKF-LSTM system is proposed that generates a pseudo-measurement of inertial velocity, feeds it to the magnetic field gradient-based EKF, and uses its dynamic to tune its corresponding covariance matrix.

\section{B. Magnetic field gradient-based EKF aided by LSTM net- work}

The implementation details and evaluation results of the proposed EKF-LSTM approach are detailed in the following, starting from the training process until the estimation one. The result of the reconstructed trajectory corresponding to the estimated velocity is also analyzed and discussed.

1) LSTM training details: The full proposed algorithm is implemented under Matlab using the deep learning toolbox. The diagram represented in Fig. 44illustrates the architecture of the designed LSTM network. It starts with a sequence

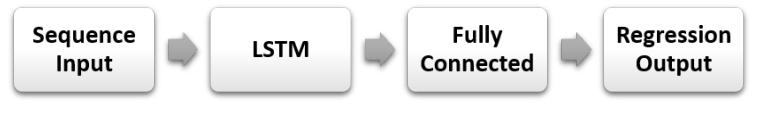

Fig. 4: Diagram of the used LSTM network

input layer receiving 9 features, representing raw data of 3axis accelerometer, 3-axis gyroscope, and 3-axis magnetometer, followed by a LSTM layer containing 100 hidden units. Then, a fully connected layer to the 3-axis velocity $v_{l s t m}$, and finally a regression layer that computes a loss function representing the Mean Squared Error (MSE) between the true velocity (Openshoe INS/ZUPT velocity in this case) and the determined one. This network computes then, for each time step $i$,

$$
v_{l s t m, i}=\operatorname{LSTM}\left(w_{i}, a_{b, i}, B_{b, i}\right)
$$

For the training process, the Adam optimizer [8] is used with an initial learning rate of 0.01 , and the model is trained for 100 epochs. Before training, a standardization [1] is applied on the Openshoe dataset, which consists basically in subtracting the arithmetic mean from the given data and dividing it by the standard deviation. Then, $50 \%$ of this data are used to form the training set and the other $50 \%$ are for the testing. The $50 \%$ training portion is equivalent to 7038 samples, which is considered small for a training process. This is the advantage of the proposed approach, that it doesn't require any complex artificial intelligence step that might need a large time computation or memory usage. The result of inertial velocity determination using the LSTM, applied on the testing set is displayed in Fig.5. As expected, the accuracy of the determined velocity from the LSTM during phases where it has large amplitudes is lower than the phases where the amplitude is close to zero. As demonstrated in Fig. 2, this is due to the usage of a training set that lacks high amplitudes velocity data.
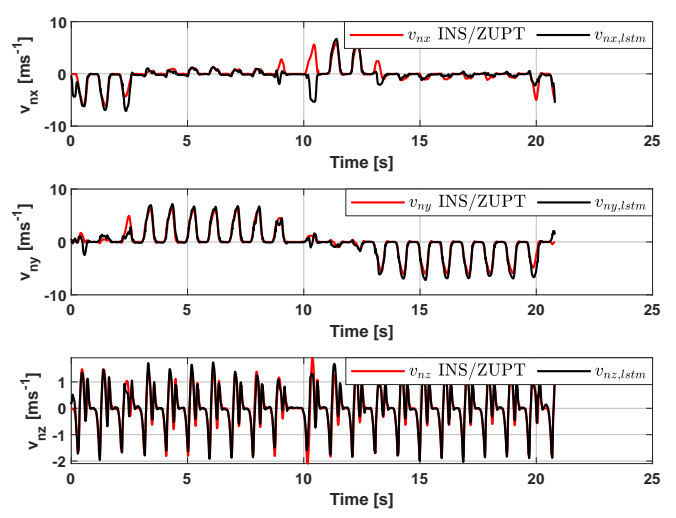

Fig. 5: Determination of $v_{n}$ with only the LSTM algorithm

Other deep learning algorithms may outperform this LSTM learning model, and adding more training data can improve accuracy results, however, as indicated earlier, a high determination accuracy is not required at this step, as the final velocity estimation is obtained from the magnetic field gradient-based EKF.

2) EKF-LSTM estimation results: The velocity estimate $v_{l s t m}$ is fed to the measurement vector of EKF (as pseudomeasurement). Within its loop, algorithm 1 is ran, to dynamically adapt the corresponding covariance matrix. The threshold $\epsilon$ is $0.1 \mathrm{~ms}^{-1}$ and the multiplication factor $\alpha$ is $10^{4}$. These values correspond to the tested data, and can change according to the studied dataset. The performance of the proposed EKF-LSTM-based estimation is evaluated on the testing set, by comparing the obtained results to the INS/ZUPT algorithm ones. In Fig. 6, the 3-axis components of inertial velocity $v_{n}$ are plotted. The velocity estimate $\widehat{v_{n}}$ (blue solid line) given by the approach is now very close to the INS/ZUPT estimate (red solid line). The RMSE between the two results is around $0.23 \mathrm{~ms}^{-1}$, which is very promising considering that the proposed method does not use any footmounted related estimation techniques.

To analyze the effect of velocity estimation on the trajectory reconstruction, $\widehat{v_{n}}$ is integrated and the $2 D$ estimated position $\widehat{M_{n}}$ is plotted and represented in Fig. 7. The estimated trajectory (blue solide line) is close to the tested one (black solid line), and the same squared shape is maintained.

Table I presents the traveled distance, as well as the coordinates of the arrival points for both trajectories. The arrival point of the tested trajectory is different from the starting point, that is the same for both trajectories.

The error of the final arrival point between the two trajectories is around $1.8 m$ which competes with top ranked methods in the literature, that require either a costly and heavy preinstalled infrastructure or a very specific sensor location (as the case for ZUPT). It is also important to highlight that in 

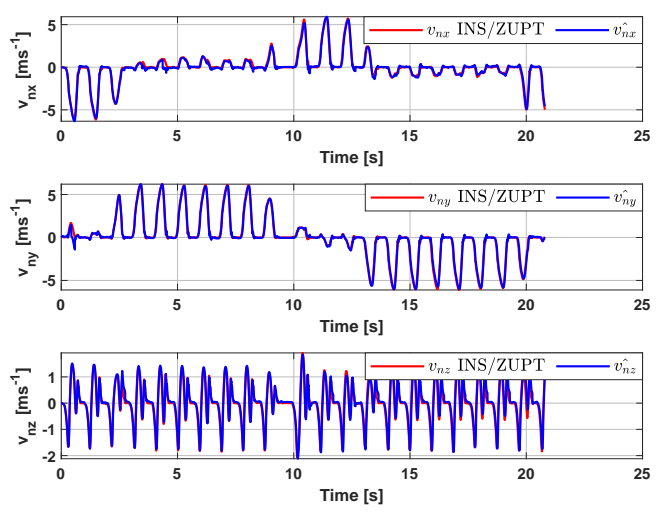

Fig. 6: Estimation of $v_{n}$ with the EKF-LSTM system

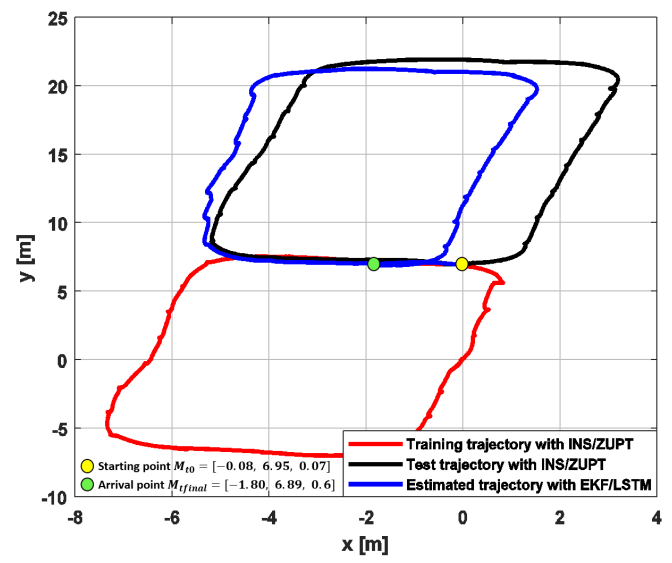

Fig. 7: $2 D$ trajectory reconstruction

TABLE I: Comparison between the INS/ZUPT reconstructed trajectory and the one from the proposed approach

\begin{tabular}{|l|l|l|}
\cline { 2 - 3 } \multicolumn{1}{c|}{} & INS/ZUPT & EKF/LSTM \\
\hline Traveled distance $[m]$ & 45.01 & 43.42 \\
\hline Arrival point coordinates $[\mathrm{m}]$ & {$[-0.41,7,0.06]$} & {$[-1.8,6.89,0.6]$} \\
\hline
\end{tabular}

this work, the Openshoe INS/ZUPT estimation results are considered as the ground truth. However, no conclusions can be made on their accuracy, as no actual ground truth is provided. This means that results given by the proposed approach, can be closer to reality than the ones computed from the INS/ZUPT approach.

\section{CONCLUSIONS}

In this paper, the inertial velocity and position estimation from a magnetic field gradient-based EKF aided by a LSTM algorithm was proposed. The LSTM model was introduced to provide a pseudo-measurement of velocity for the EKF, and a covariance matrix adapter was applied to dynamically tune its corresponding noise parameters. The final inertial velocity estimate from the proposed approach competes with the INS/ZUPT results, as it requires only IMU and magnetic measurements, and doesn't rely on any particular sensor location or walking pace parameterization. It was shown that the reconstructed trajectory from this novel method is very close to the one from INS/ZUPT technique, and small drifts were observed. Other datasets corresponding to different applications (e.g. drones, land robots, etc.) need to be tested to highlight more the efficiency of the proposed solution.

\section{REFERENCES}

[1] H. Anysz, A. Zbiciak, and N. Ibadov. The influence of input data standardization method on prediction accuracy of artificial neural networks. Procedia Engineering, 153:66-70, 2016.

[2] E.-R. Bachmann, X. Yun, and C.-W. Peterson. An investigation of the effects of magnetic variations on inertial/magnetic orientation sensors. In International Conference on Robotics and Automation, pages 11151122, New Orleans, LA, USA, 2004.

[3] M. Brossard, A. Barrau, and S. Bonnabel. AI-IMU dead-reckoning. ArXiv, 2019.

[4] C.-I. Chesneau, M. Hillion, J.-F. Hullo, G. Thibault, and C. Prieur. Improving magneto-inertial attitude and position estimation by means of a magnetic heading observer. In International Conference on Indoor Positioning and Indoor Navigation (IPIN), pages 1-8, Sapporo, Japan, 2017.

[5] H. Fourati. Heterogeneous data fusion algorithm for pedestrian navigation via foot-mounted inertial measurement unit and complementary filter. IEEE Transactions on Instrumentation and Measurement, 64(1):221-229, 2015.

[6] S. Hochreiter and J. Schmidhuber. GLong short-term memory. Neural Computation, 9(8):1735-1780, 1997.

[7] J.D. Jackson. Classical Electrodynamics. Third Edition. John Wiley \& Sons, Inc., 1998.

[8] D. P. Kingma and J. Ba. Adam: A method for stochastic optimization. In International Conference on Learning Representations, San Diego, USA, 2015.

[9] J.B. Kuipers. Quaternions and rotation sequences. 1999.

[10] M. Mahmoud, I. Alaa, A. Wassal, A. Noureldin, and A. Eldieb. Tuning of the error covariance parameters in EKF-based INS/GPS systems: A practical approach. In International Conference on Mobile Mapping Technology (MMT), Cairo, Egypt, 2017.

[11] T. Michel, P. Genevès, H. Fourati, and N. Layaïda. Attitude estimation for indoor navigation and augmented reality with smartphones. Pervasive and Mobile Computing, 46:96-121, 2018.

[12] M. Sazli. A brief review of feed-forward neural networks. Communications, Faculty Of Science, University of Ankara, 50(1):11-17, 2006

[13] I. Skog, J.-O. Nilsson, and P. Händel. Evaluation of zero-velocity detectors for foot-mounted inertial navigation systems. In International Conference on Indoor Positioning and Indoor Navigation (IPIN), pages 1-6, Zurich, Switzerland, 2010.

[14] I. Skog, J.-O. Nilsson, P. Händel, and K.V.S. Hari. Foot-mounted INS for everybody - an open-source embedded implementation. In Position Location and Navigation Symposium (PLANS), pages 140-145, Myrtle Beach, SC, USA, 2012.

[15] D. Vissière, A.P. Martin, and N. Petit. Using magnetic disturbances to improve IMU-based position estimation. In European Control Conference, pages 2853-2858, Kos, Greece, 2007.

[16] D. Vissière, A.P. Martin, and N. Petit. Using spatially distributed magnetometers to increase IMU-based velocity estimation in perturbed areas. In Conference on Decision and Control, pages 4924-4931, New Orleans, Louisiana, USA, 2007.

[17] D. Williamson, RA. Parker, and J. Kendrick. The box plot: A simple visual method to interpret data. Annals of internal medicine, 110(11):916-921, 1989.

[18] M. Zmitri, H. Fourati, and C. Prieur. Improving inertial velocity estimation through magnetic field gradient-based extended kalman filter. In International Conference on Indoor Positioning and Indoor Navigation (IPIN), pages 1-7, Pise, Italy, 2019. 